\title{
ADEVA: A Decentralized Electronic Voting Application Using Blockchain Technology
}

This paper was downloaded from TechRxiv (https://www.techrxiv.org).

\section{LICENSE}

CC BY 4.0

SUBMISSION DATE / POSTED DATE

$13-01-2022$ / 19-01-2022

CITATION

ABEGUNDE, JACOB (2022): ADEVA: A Decentralized Electronic Voting Application Using Blockchain Technology. TechRxiv. Preprint. https://doi.org/10.36227/techrxiv.18331538.v1

DOI

10.36227/techrxiv.18331538.v1 


\title{
ADEVA: A Decentralized Electronic Voting Application Using Blockchain Technology
}

\author{
Jacob Abegunde and Joseph Spring, University of Hertfordshire UK \\ Hannan Xiao, King's College London UK
}

\begin{abstract}
Despite the various advantages inherent in the use of blockchain technology, blockchain application platforms are characterized by bottlenecks, latency, energy inefficiency, time inefficiency, low transaction throughput, vulnerability to $51 \%$ attacks, and a lack of fairness in the sharing of mining proceeds. These challenges are currently affecting the performance of this technology, hindering its rapid development and adoption for real-time applications such as Electronic Voting Applications (EVAs). It is well known that the consensus mechanism responsible for the security and consistency features of a blockchain, is also responsible for most of the above challenges.

In this paper, a new consensus mechanism, Proof of Vote $(\mathrm{PoV})$ is proposed, a lightweight consensus mechanism specifically adapted for EVA, as a replacement for the heavyweight Proof of Work (PoW) consensus algorithm. We use the Ethereum blockchain as a decentralized application platform, in which we develop a decentralized electronic voting application (ADEVA), replacing the Ethereum PoW consensus algorithm with the proposed PoV consensus algorithm. Thus we implemented ADEVA with PoV and compared it to the two major consensus mechanisms: Proof of work (PoW) and Proof of Stake (PoS). We found that PoV enhanced the performance of ADEVA over and above that achieved using PoW and PoS. The low resource utilization and high transaction throughput of ADEVA with PoV makes it a scalable solution for real-world EVAs.
\end{abstract}

Index Terms-Decentralized Application, Distributed Security, Trustless System, Blockchain, Smart Contract, E-voting.

\section{INTRODUCTION}

$\mathbf{T}$ He traditional paper-based voting has a number of challenges which are location-based, accuracy based, scalability based or cost [1], [2]. Electronic Voting Applications (EVA) have been proposed as an alternative to paper based voting because of its potential to overcome the above challenges [3], [4]. This makes EVA more appealing. However, the majority of existing EVA's do not satisfy all of the requirements of an EVA such as 'universal verifiability and receipt freeness', 'transparency and privacy', robustness, tamper-proof, and resilience. [1], [5].

Most previous implementation of EVA are based on a clientserver architecture with a centralized single database system [6], [7]. This single database system, could at best, serve as a single point of interest for the developer, a single point of control and a single point to secure. However, it could also serve as a single point of attack for the adversary and consequently a Single Point of Failure (SPF).

These vulnerabilities, coupled with recent advancement in knowledge and technology have shown that such applications are inadequate [8], [9], especially as transparency and security are paramount for public trust and acceptance of an EVA, There is therefore a need for an alternative solution.
Some of the requirements of EVA such as 'confidentiality versus transparency', 'verifiability versus receipt-freeness', are seemly incompatible [10], [11], which make the process of designing an EVA a challenging task. As a result of this, although many EVAs have been proposed and developed to address these challenges, EVA's remains an open area of research.

The proof of concept of blockchain technology has been demonstrated by its successful implementation in Bitcoin cryptocurrency [12], [13]. The features of blockchain technology such as decentralization, transparency, traceability, and tamper resistance makes it a suitable technology for EVA's and many of them have been proposed and implemented [14], [15].

However, for real world scenarios, blockchain is not a panacea for all application development because it has it own drawbacks such as bottlenecks, ultra-high energy consumption, latency, and vulnerability to $51 \%$ attacks [12], [16]. These drawbacks need to be taken into consideration when developing real-time application such as an EVA on a blockchain platform.

In this work, we examined the consensus mechanism which is the core security module of blockchain technology and considered its potential for optimisation with EVA's in terms of energy consumption, transaction throughput, latency, and convergence time without sacrificing security.

Our simulation results show that the proposed consensus algorithm, Proof of Vote (PoV), achieves faster convergence, better transaction throughput, higher transaction speed, lower latency, and better energy efficiency than the existing algorithms whilst still meeting the requirements of EVA.

\section{A. Motivation}

The challenges faced by paper based ballot systems [17], [18], the vulnerabilities experienced by current EVAs [1], [17], recent advances in technology [9], [19] coupled with the proliferation of smart devices and protocols [6], [20] suggest the need for a new EVA to be developed. The principal motivation for our work is the need to develop a trusted EVA as a potential replacement for existing EVAs. The desired features for our proposed EVA are: authentication of voters, immutability of code and votes, robustness and resilience to failure and DoS attack, auditability and verifiability of code and votes, resistance to interference, fraudulent alteration, tallying error and counting error.

The proven security features of blockchain technology as demonstrated in bitcoin cryptocurrency is another motivating influence for this work. In other words, the successful implementation of blockchain technology in bitcoin cryptocurrency 
as evidenced in [12], [21] suggest the plausibility of pursuing the application of this technology to the challenges of security and trust with EVA's.

\section{B. Contribution}

In this work, our main contributions are threefold.

1. An EVA is proposed and designed using the concept of a decentralized system architecture. Our application is developed on an Ethereum blockchain to protect the application code and data from Denial of Service (DoS) attacks, Single Point of Failure (SPF), and fraudulent modification of record. It also includes Multi-Factor Authentication (MFA) and One Time Password (OTP) to protect voters from risk associated with stolen credentials.

2. Although the security of blockchains have been partly attributed to the PoW consensus, the same PoW consensus has been criticized as being responsible for latency, bottlenecks, and energy inefficiency [12], [13]. The most popular alternative to the PoW consensus is the Proof of Stake (PoS) consensus, however, this mechanism can lead to a monopoly effect since its mining process is based on the highest stakeholder node [12], [13]. We therefore took the position that these two consensus mechanisms are unsuitable for EVAs, and proposed, and implemented a new consensus mechanism for ADEVA, the Proof of Vote (PoV).

3. This research differs from other related work in that votes are automatically tallied and counted on the fly as they are cast. In our application, this is a non-stop process until the end of the election period after which the result is released after the last vote is cast. It follows that there is no coolingoff period during which votes may be manipulated. In most of the previously proposed EVA's [22], [23], votes are not counted as they are cast but are encrypted and stored on disk during the voting window. After the voting period, the secret key is then released for decryption, tallying, and counting of the votes.

The reminder of this paper is structured as follows. In section 2 we discuss the relevant background relating to the study followed by our proposal and model in section 3 . In section 4 we discuss the system implementation followed by system evaluation in section 5 . In section 6 we discuss the features of our model, followed by the conclusion and future work in section 7 .

\section{BACKGROUND}

\section{A. Problem Statement}

According to [23], [24], It is generally believed that the privacy and security of an electronic vote can be preserved in two ways. Firstly, by encrypting and decrypting the vote and secondly by sending the vote through an anonymous communication channel. Although both methods are good theoretically, they do not offer any guarantee of protection for the voting information, neither do they meet the tamperproof requirement of an EVA. This is especially true in the face of well-resourced adversary who has the capability and computing resources required to sniff anonymous channels and decrypt data sent on communication channels [25], [26].

The solution, therefore involve more than these two techniques of 'encrypting the vote' and 'sending it through an anonymous communication channel'. In order to address these challenges, the work in [24], was presented as a generalpurpose protocol for e-voting and it was followed by an improved version [23] with a self-tallying function, however the improved version has its own drawback such as lack of robustness and fairness as identified in [27].

Similarly, the work in [27] and [28] was released as a robust and fair solution while [23] is meant to offer an End-to-End (E2E) verification feature. However, these solutions are neither decentralized nor tamper-proof which means an adversary could still stage a DoS attack against these applications or tamper with the record of votes.

\section{B. The Proposed Solution}

An alternative solution to the above problem is to develop a decentralized application consisting of peer-to-peer nodes forming a blockchain network to share the responsibility of maintaining the network data and code integrity [7], [29]. In a fully decentralized application model, each of the nodes in the blockchain network contains the code and the data [30], [31] as illustrated in Fig. 1.
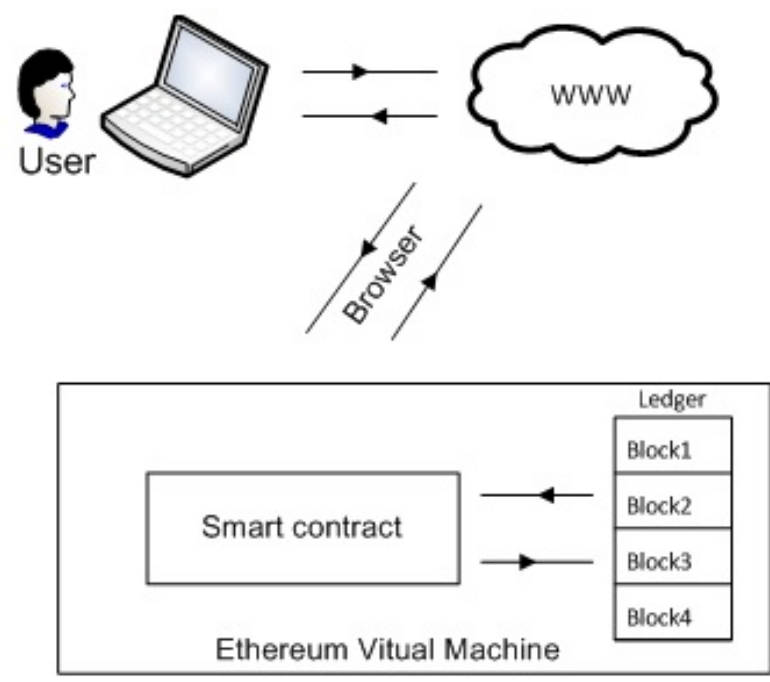

Fig. 1: A fully decentralized application node

Recent work for example [6], [32] and [33] present partially decentralized e-voting solutions in which blockchains are used as a storage media for votes whilst the application code is hosted separately. Although these solutions can protect the votes against fraudulent modification, as a result of the tamper resistance feature of the blockchain platform, the application code remains vulnerable to DoS attack and Single Point of Failure (SPF) [26], [34].

Research in [33], [35]-[37] present fully decentralised solutions that run on a blockchain platform, utilising smart contracts functionalities. One of the powerful features of this 
architecture is that the ledger record and the application code (Smart Contract) are immutable, meaning that none of the nodes can unilaterally make any change to the data or the code [32], [38]. The ledger records are arranged into blocks and chained together using a hash function to form a public record [6], [39]. This arrangement ensures that the data and the code are held securely. Since the voting record and code of an EVA are supposed to be tamper-proof, this model is more suitable for the deployment of an EVA [5], [40].

However, despite the security enhancement of a fully decentralised EVA as discussed above, the performance and the scalability of such a solution as a real-time EVA are still unknown [13], [41]. It is common knowledge that blockchain applications that use the PoW consensus mechanism are characterised by low transaction throughput, high latency and slow convergence time, communication bottlenecks, ultra-high energy consumption, time inefficiency and vulnerability to $51 \%$ attacks [13], [32].

This implies that for a blockchain to be used as a platform for real-time EVA's, there is a need to provide mitigations for these vulnerabilities and challenges particularly scalability and sustainability. It is one thing for a blockchain based EVA to successfully handle an election involving a moderate number of voters, for example one to two million, it is quite another thing for the same EVA to handle an election for a large number of voters, for example one to two billion.

The PoW consensus mechanism of a blockchain is known to lack scalability and sustainability [13]. It lacks scalability since it takes ten minutes for the network to confirm a transaction and it is only capable of handling about seven transactions per second according to Bitcoin record [12], [42]. Such latency will pose a significant challenge to EVA's particularly for large number of voters. The substantiality problem has to do with the cost of energy and carbon footprint associated with PoW [6], [32].

The use of PoS for EVA's implies that a mining node that has the largest stake will always have the privilege to package transactions into blocks (mining). This is not only nonrepresentative, it will also constitutes unfairness, especially when there is a stake or reward associated with the mining process [6], [13].

We therefore, conjecture that neither PoW nor PoS in their standard form is suitable as a consensus mechanism for EVA's. Consequently, we propose and implement a new consensus mechanism, PoV, a modified version of PoS, specifically optimised for EVA's.

In addition to the vulnerabilities to DoS attacks, SPF and record modification, as discussed above, another challenge for most current EVAs is the 'tallying and counting' problem and the time it takes to release the election result [22], [43]. It is common knowledge that the longer it takes to release an election result, the less the public will trust the outcome. In order to solve these problems, most of the previously proposed self-tallying EVA's encrypt and store votes on disk for tallying and counting after the voting window expires [22], [44]. This implies that there is a cooling-off period in which votes are vulnerable to manipulation before they are tallied and counted. We therefore we propose a self-tallying EVA in which votes are tallied and counted on the fly as they are cast and the result is released immediately after the last vote is cast, in order to ensure credibility and trustworthiness with respect to the election result.

\section{SYSTEM DESIGN}

In order to describe our ADEVA model, we describe terminologies, and made some assumptions below.

\section{A. Terminology Definition}

The following terms as used in this discussion.

- Voters: This is the list of people who are eligible to vote.

- Candidates: This is the list of contestants.

- Electoral Commission (EC): This refers to the election authority.

- Blockchain admins: These are EC system administrators.

- Political parties: This refers to 3 political parties: Blue, Green and Red.

- Local Authority (LC): This is the constituency in which election is being held.

- Blockchain network: A non-trusted peer-to-peer network of nodes that maintains ledger records.

- Mining nodes: A set of nodes that are responsible for updating the blockchain ledger.

- Non-mining nodes: A set of nodes whose role is to observe and verify transactions.

- Smart contracts: A set of software code on a blockchain that manages the ledger.

\section{B. Model Assumptions}

The assumptions of the proposed ADEVA are as follows:

- Rationality: All actors, both good and bad, are rational. The good actors will try to maximize their benefits whilst adhering to the rules. The bad actors will try to maximize their revenue through legitimate and illegitimate means, but they will not launch any attack that is against their own interest.

- Unpredictable behaviour: The behaviour of bad actors are considered as unpredictable. They may operate honestly to profit themselves or act dishonestly to profit themselves individually or collusively.

- Honest majority: We consider a consortium blockchain which includes three political parties, the electoral commission, the local authority and various observers. In such a scenario, it is reasonable to assume that the number of honest actors will always exceed the number of malicious actors. Collusion is considered as highly unlikely due to a conflict of interest.

- Irreversible hash function: Hash functions are considered to be irreversible, which means that while a hash value could be computed for a message, the original message cannot deduced from a given hash value. In addition to this, it is also assumed that a repeated hash of the same input message will certainly yield the same hash 
value, and hence the probability of two message inputs generating the same hash output is unlikely and hence insignificant.

- Secret keys: A secret key of 24 characters length is auto-generated for voters and nodes using the Rivest-Shamir-Adleman (RSA) algorithm. It is assumed that these secret keys are sufficiently secure at least for the duration of the voting window. The task of breaking them within the 24 hour voting period is considered to be non-trivial. This implies that no malicious actor can impersonate another actor during the voting window because of the unknown private key.

- Communication: It is assumed that any message sent by any system actor will arrive at its destination within a finite time interval, i.e TCP retransmission mechanism will resend lost or corrupted messages until the delivery is successful.

- Winning criteria: The candidate who scores the highest number of votes in a constituency is considered as the winner in the constituency.

- Voters database: It is assumed that the EC has an accurate database of registered voters which will be written to the blockchain and will be updated regularly.

\section{ADEVA Model}

As shown in Fig. 2, the ADEVA model is built on an Ethereum blockchain platform. It consists of a user interface, the smart contract, and the ledger record, which are all hosted on the Ethereum Virtual Machine (EVM), a blockchain infrastructure on a peer-to-peer network of nodes [32], [45]. Intercommunication between nodes is via encrypted multicast or broadcast. The votes are stored in bundles of records called blocks, which are chained together using a hash function to form a ledger [33], [46].

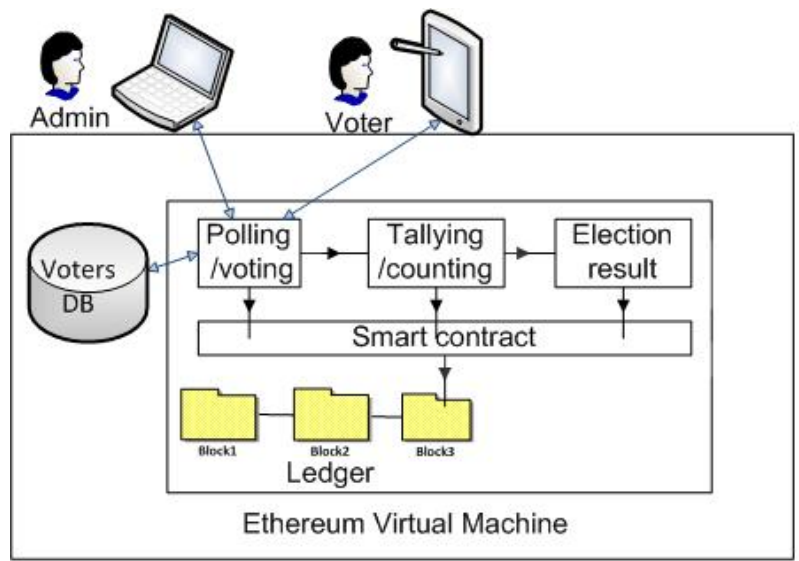

Fig. 2: ADEVA Architecture

All the data in the ledger are secured by the immutable features of the blockchain [47], [48]. The frontend user interface consist of the administrative user interface and the voters' user interface. The administrative user interface consists of nodejs which enables the deployment of the smart contract on the nodes. The voters' interface is coded into the smart contracts which present the election functionalities to voters. Therefore the ADEVA application is hosted on a network of nodes, in which each node contains the user interface, the smart contract, and the ledger where the votes are stored. The smart contract presents the application functionalities to users and also modifies and manages the ledger record (votes).

\section{ADEVA Phases}

The three phases of ADEVA are:

The Registration Phase: The registration of voters is an identity check process to determine the eligibility of voters to vote. It serves as input to the election and it normally ends before the commencement of the election. Rather than having a centralized system, the electoral process is divided into constituencies. This implies that registration of voters is based on constituency, likewise the deployment of the ADEVA and the voting process. The voters' eligibility check is based on the registration record.

The Voting Phase: The voting phase is subdivided into: Initialization, MFA, and Voting Process.

- The initialization process refers to the closing of the voters' registration process followed by the deployment and publication of the ADEVA in readiness for the election. This is the responsibility of system administrator in the EC.

- The MFA constitutes an identity verification process in which the information provided by voters during the registration process are verified. This information includes a preset voter identification and password. In the first stage of the MFA the voter supplies their voting identification and password for verification. If the verification is successful, a One Time Password (OTP) is sent to the voter's known mobile number, provided during the registration process. This OTP needs to be typed into the application for MFA to be successful.

- A successful MFA is a prerequisite to voting. That is, voters are presented with the voting interface if and only if their MFA is successful. The voting menu consist of a drop-down list of the candidates to be voted for and a 'vote' button below it. Voters will select a candidate from the list and select 'vote' button to cast their vote.

The Tallying and Counting Phase: The tallying process refers to the separation of votes according to the party of the candidate of choice whilst the counting refers to the summation of votes for each party or candidate. In the ADEVA simulation, the tallying and counting processes are carried out automatically on the fly by the smart contract, without human intervention. This is to eliminate human error and reduce the waiting time for the election result.

\section{E. ADEVA Algorithm}

Fig. 3 shows the ADEVA flowchart. The algorithm is outlined below:

- Step 1: Application initialization and publication. 


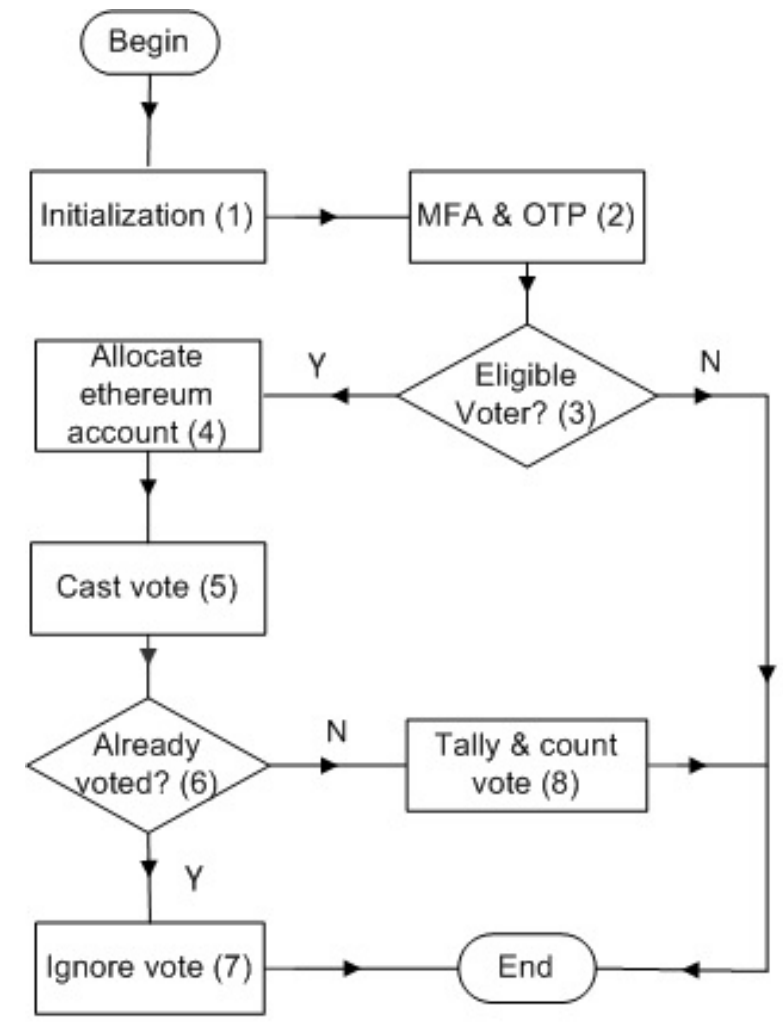

Fig. 3: ADEVA Flowchart

- Step 2: Voters' authentication (MFA and OTP).

- Step 3: Voter's eligibility check against registration list.

- Step 4: If steps 2 and 3, then allocate a voting account.

- Step 5: Allow casting of vote

- Step 6 \& 7: Ignore vote if voter has voted earlier.

- Step 8: Tally, count and record votes.

\section{F. Consensus Mechanisms}

The core module of the blockchain technology, responsible for determining the conduct of the mining process, the security and the consistency in the ledger record is the consensus mechanism. The performance of a blockchain application is attributed to the performance of the consensus algorithm [13], [49], in other words, the consensus mechanism affects the performance metrics such as the energy cost, time efficiency, transaction throughput, and latency of the blockchain application.

The security and tamper-proof features of a blockchain application is also attributed to the fact that a hash value is generated for every transaction contained in the block and a unique block hash value is calculated from the individual transaction hash values. In the Bitcoin implementation, the block hash value, the time stamp, the hash value of the previous block and a nonce generated through PoW are all written in the block header. Thus, any modification in the transactions or the block header, will lead to a different block hash value which will subsequently lead to the recalculating and rewriting of all the subsequent blocks [12], [13].

The complexity of this mathematical puzzle of PoW is further enhanced by the fact (or assumption) that a hash function is irreversible. Under such circumstances, any actor who wants to tamper with a block transaction will have to re-choose all the nonce values correctly and recalculate all the hash values of all blocks from the target block to the end of the blockchain since each block header contains the hash value of the previous block. This puzzle and work must be done at a speed faster than the speed of adding legitimate records to the longest chain [50], [51].

At the moment, such a recalculation and rewriting are believed to be non-trivial tasks, although the continuos advancement in technology could make this a trivial task in the future. In the likelihood that it turns out to be a trivial task for, for example a sophisticated device such as a quantum computer, when the rewriting of the transactions and the blocks are completed, the honest nodes will still reject such a bogus ledger [52], [53].

Hence whenever there is an honest majority nodes, there will be no motivation for an actor to engage on such nontrivial tasks since it will effectively be a waste of resources and time when the fraudulent ledger is rejected by the honest majority. Therefore, from the security point of view, PoW is considered invincible. However, the PoW consensus comes at the cost of resource utilization, latency, low system throughput, and consequently a low quality of service which makes it unsuitable for real-time application such as EVA [13], [54].

In Bitcoin implementations, in order to reduce inconsistency in the ledger, the block interval is set to an average of 10 minutes to reduce the probability of forking [13]. In addition, each transaction on the block needs to wait for the mining of six subsequent blocks before they can reach a final commit state in order to prevent double spending attacks [12], [13]. This will not meet the real-time requirements of EVA.

As as result of the challenges of the PoW mechanism, the development of an efficient consensus mechanism has been an ongoing area of research for blockchain applications. This has led to the proposal of various consensus mechanism, the most prominent among them being, the Proof of Stake (PoS) which is considered a viable alternative to PoW. In the PoS consensus, the miner of a block is randomly chosen based on the stake owned by the participant. This implies that the miner with the largest stake is always chosen since it is rational for it to protect its stake by complying with the rule. In other words, a dishonest action on its part will destroy its own stake.

However, in both the PoW and PoS mechanism, the privilege to mine a block is proportional to the CPU power and the stake of the nodes respectively. This implies that only the nodes with high CPU or stake will have the privilege of mining blocks and earning rewards, thus leading to unfairness in the sharing of mining proceeds [55].

In order to mitigate these shortcomings, a new consensus algorithm, the PoV is proposed for ADEVA to improve performance in terms of time efficiency, energy efficiency, transaction throughput, resources utilization, fairness and the security of the mining process. 


\section{G. Proof of Vote (PoV)}

The flowchart for the PoV is as shown in Fig. 4. To the best of our knowledge, PoV is specific to ADEVA and it has never been used anywhere.

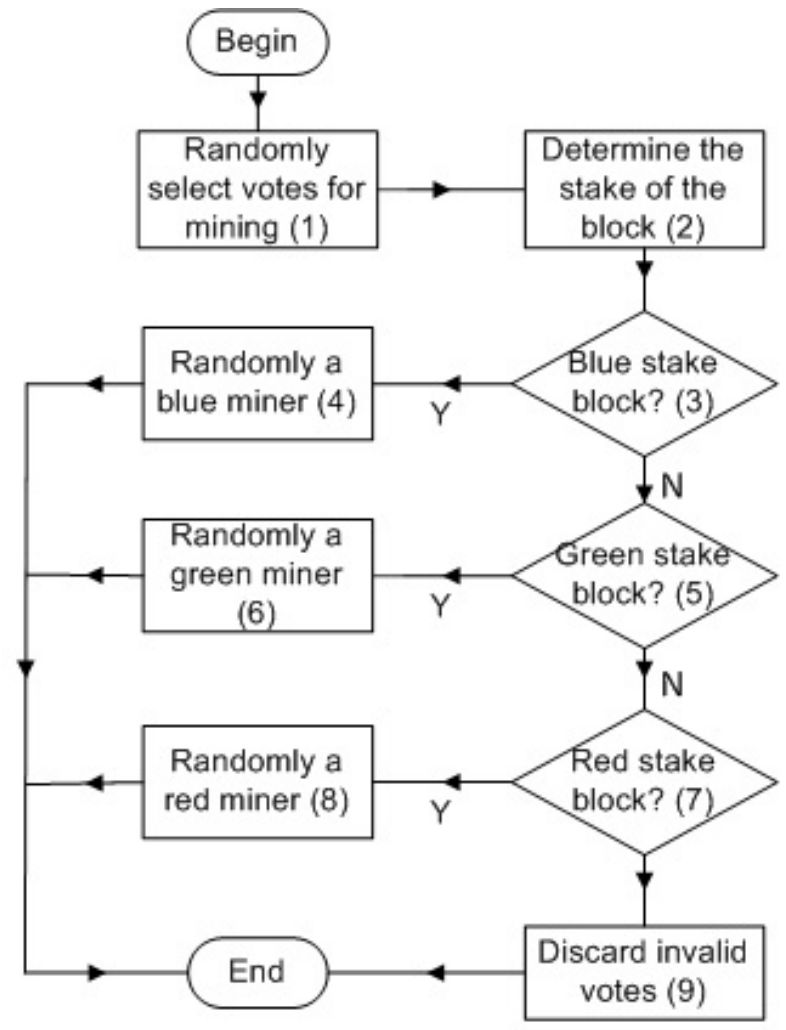

Fig. 4: PoV Flowchart

In the PoV mechanism, the mining privilege is allocated, based on the stake of each block. The cast votes are selected and arranged into blocks in the order of first come first served (FCFS). The number of votes in a block is set as optional parameter consisting of values 10, 20, 30, etc in the configuration file. The value of the stake of a block is calculated as a probability value of the party or candidate of choice. The stake is allocated to the party that has the highest probability value. For instance, a blue stake is assigned to a block with a higher number of blue votes than red votes and green votes. The same is applicable to a green stake block and a red stake block.

The implication of this is that the mining privilege for any block is assigned to the nodes of the political party that won the majority of the votes in that block since they are the largest stakeholder for that block. For each block of votes, the mining node is then randomly selected from the mining nodes of the political party that won the majority of the votes in that block in order to prevent DoS. In other words, for a blue stake block to be mined, the miner is randomly selected from the list of blue miners.

We refer to a block as single stake if there emerges a single party that scores the highest number of votes in the block, while a double stake block refers to a block in which there exist two winners, and triple stake refers to a block in which all the three political parties have the same number of votes. For a double stake block, the miner is randomly selected from the combined miners of the two political parties that won the largest number of votes in the block. The same principle is applicable to the triple stake block.

The PoV is a modified version of PoS which is fine-tuned for ADEVA. It is designed not only to remove the energy wastage in PoW, but to also reduce the convergence time, improve transaction throughput and consequently reduce the latency in the application. The workflow for the proposed PoV is as shown in Fig. 4 and the algorithm is outlined below:

- Step 1: Select votes from the transaction pool in to a block.

- Step 2: Compute the stake of each party on the block

- Step 3\&4: Select Blue miner to mine Blue stake block.

- Step 5\&6: Select Green miner to mine Green stake block.

- Step 7\&8: Select Red miner to mine Red stake block.

- Step 9: Discard invalid votes.

\section{IMPLEMENTATION}

\section{A. Experimental Setup}

In order to develop and implement a decentralized application on an Ethereum Virtual Machine (EVM), an Ethereum Integrated Development Environment (IDE) is required to simulate blockchain nodes and their interaction or message exchange [33].

The following open-source software was installed and linked together to form the blockchain simulation environment.

- Ubuntu Linux: Ubuntu Linux is used as the base operating system

- Nodejs: Node.js is for application distribution across Ethereum nodes [56].

- NPM: Network package manager is for the administration of Nodejs [56].

- Solidity Compiler: Solidity is a programming language for the writing of smart contracts on Ethereum [57].

- Ganache: Ganache provides a means for simulating personal blockchain accounts [11].

- Truffle Framework: Truffle enables testing, compilation, and deployment of smart contract code [29].

After the installation, configuration and testing of this IDE, it is used in the writing, testing, and deployment of smart contracts for ADEVA.

\section{B. ADEVA Simulation}

Fig. 5a shows the initial experimental setup. A consortium model for the blockchain is considered which is a permission blockchain in which there are six main stakeholders: the three political parties (Blue, Green and Red), the Electoral Commission (EC), Local Authority (LA), and the public observers. The three political parties are considered as the largest stakeholders and the same stake is assigned to each of them for equal representation. Thus, each party is assigned the same number of mining nodes.

For the initial experimental setup, three mining nodes: B1, G1, R1 are assigned to the Blue, Green, and Red political 


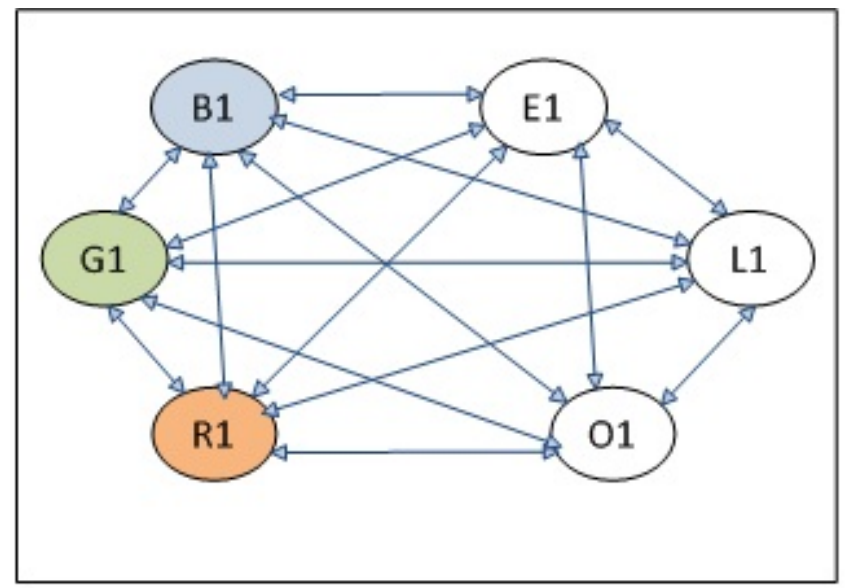

(a) ADEVA Nodes Simulation

\begin{tabular}{|c|c|c|}
\hline $\begin{array}{c}\text { Hash Value } \\
\text { Blue Vote } \\
\text { Time: } 15 / 02 / 21: 16: 20: 10 \\
\text { TIN: xxxxxxxxxxxxxxxxx } \\
\text { Blue Party } \\
\text { Blue Candidate } \\
\text { Ballot ID }\end{array}$ & \begin{tabular}{|c|} 
Hash Value \\
Green Vote \\
Time: $15 / 02 / 21: 16: 22: 05$ \\
TIN: xxxxxxxxxxxxxxxxxx \\
Green Party: \\
Green Candidate \\
Ballot ID
\end{tabular} & $\begin{array}{c}\text { Hash Value } \\
\text { Red Vote } \\
\text { Time: } 15 / 02 / 21: 16: 25: 15 \\
\text { TIN: xxxxxxxxxxxxxxxxxx } \\
\text { Red Party: } \\
\text { Red Candidate } \\
\text { Ballot ID }\end{array}$ \\
\hline
\end{tabular}

(b) ADEVA Vote Information

Fig. 5: ADEVA Node and Vote

parties respectively, whilst three non-mining nodes: E1, L1, O1 are assigned to EC, LA and public observers respectively, these nodes are not major stake holders and hence are not allowed to package votes into blocks (mining). They are classified as monitoring, verifiers and observer nodes. The number of the mining nodes for each political party could be increased by a factor of 2, 3, 4, and so on, however, the three political parties must have an equal number of nodes at any point in time for fairness reason. The number of the monitoring and observer nodes could be any positive integer.

The structure of the electronic vote is as shown Fig. 5b. Each vote on the ledger is an encrypted record consisting of timestamp, Transaction Identification Number (TIN), the candidate or party of choice and a hash value of the vote. The votes are also classified as Blue vote, Green vote or Red vote based on the party or candidate of choice.

ADEVA is designed to allow only one vote per eligible voter and a choice of only one candidate or party. It is possible for voters to waste their votes by not voting for any candidate. They can achieve this by logging out without choosing a candidate. However, voters cannot vote for two or more candidates because they can only choose a candidate from the option list, neither can they change their vote since ADEVA is designed to tally and count the first vote only and ignore subsequent votes by considering them as a double spending attempt. The application assigns a single voting account to each eligible voter based on a valid login credential and it is designed to track and prohibit double spending (voting) by crediting each voting account with an Ethereum coin which can be spent once in the voting process.

The three mining node addresses and authentication requirements are set up in the configuration files. The total number of simulated nodes in this first simulation run was six (as shown in Fig. 5a) and the number of voting accounts generated were 10 (as shown in Fig. 6b). These parameters were also setup in the configuration files, the smart contracts were deployed and the web address and service port of the application are published using the truffle framework. After this deployment, voters are able to access the user interface (voting menu) using their browsers. Fig. 6a, shows the voting interface while Fig. $6 \mathrm{~b}$ shows the back end audit $\log$.

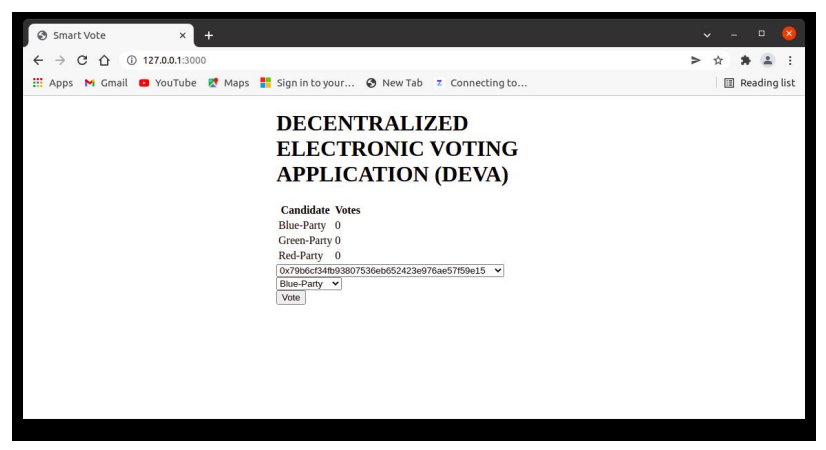

(a) User interface showing the voting menu

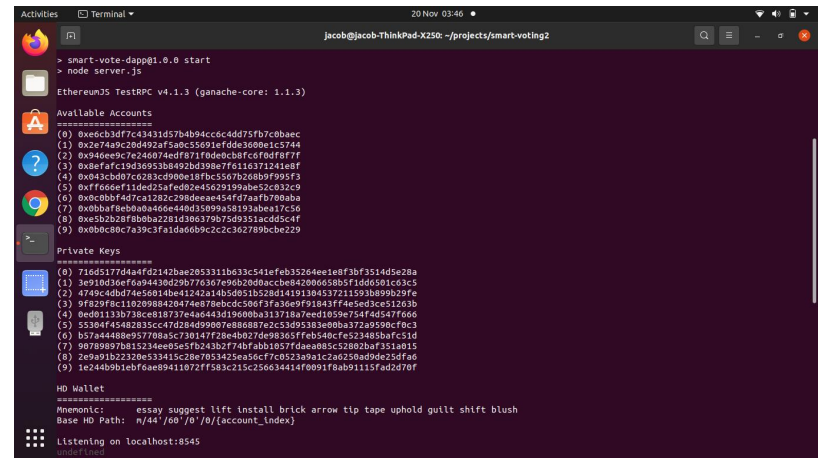

(b) Transaction audit logs showing user activities

Fig. 6: ADEVA voting interface and audit logs

The voting interface in Fig. 6a was used to cast ten votes based on the ten voting accounts generated, since each account can only vote once. The voting starts with the user's authentication request which includes the process of MFA and eligibility the check. If the MFA and eligibility check are successful, a voting account is randomly allocated to anonymize the voter who is presented with the voting menu in order to caste their vote. After the vote has been cast, the allocated coin for the voting account is spent and will no longer be available for spending. Hence subsequent votes for the same account will be ignored.

The information contained in a vote is as shown in Fig 5 b. It contains no voter's identification information. The vote is hashed and the hash value is stored before the vote is encrypted. The encrypted vote and their hash values are kept in the memory pool for mining while the generated Transaction Identification Number (TIN) and the hash value of the encrypted vote are stored in the application for future verification purposes. After the voting, the counting and the mining are completed, the unique TIN and the block reference 
number ensures that individual encrypted votes can still be located as a transaction in the blockchain ledger. The current hash value of the transaction (vote) can be compared with its original hash value to prove that the vote has not been tampered with.

\section{SYSTEM EVALUATION}

\section{A. Accuracy and Transparency}

In order to evaluate the accuracy and transparency of ADEVA, at the end of the initial run of the protocol with 6 mining nodes and 10 voters, the vote count was examined to ensure that it corresponded with the votes cast at the user interface (Fig. 6a) and the audit log record (Fig. 6b). The back end was also examined to ensure that it agreed with events carried out at the user interface. The three event variables were compared and they were in agreement. The vote cast on the user interface, the displayed votes-count on the report interface and the audit log at the back end, all matched one another verifying that votes were tallied, counted and recorded as cast, a demonstration of accuracy and transparency.

\section{B. Anonymity and Coercion-free}

Fig. 6a and Fig. 6b show the voting interface for voters together with thee back end audit logs. These contain no information relating to voter identification. Likewise, the vote information (Fig. 5b), written into the ledger by the smart contract is as shown in the audit log (Fig. 6b). This combination shows that a voter's information is dissociated from their votes, being replaced with the voting account as expected. The votes could be verified after the election using the TIN and the hash value of the vote. However, the absence of a link between voters' identification and their vote, as shown in Fig. $5 \mathrm{~b}$ will make it difficult for voters to prove how they have voted to a coercer for, for example, reward. The verification process compares the hash value of the original vote with their current hash value to prove that the votes have not been altered. It does not confirm the candidate or the party that the voter has voted for. There is therefore, no motivation to coerce.

\section{Resources Utilization and Transaction}

Similar to the Bitcoin crypto currency platform, the native or default consensus for the Ethereum blockchain is PoW whose challenges have been discussed earlier. In order to investigate the performance of the PoV consensus, a total of thirty mining nodes (ten nodes for each political party) and twenty million voters was employed in simulating ADEVA with PoW and PoS, the most popular consensuses in current use and compared their performance with performance of ADEVA with PoV. The resulting resource utilization for PoW, $\mathrm{PoS}$ and PoV are as shown in Fig. 7a.

The result of our simulation shows a lower resource utilization for ADEVA with PoV than for ADEVA with PoW. We attributed this to the removal of the heavyweight mathematical puzzle associated with PoW. The utilization for ADEVA with PoS was better than ADEVA with PoW, as expected for the same reason. Similarly, the PoV compete favourably with PoS

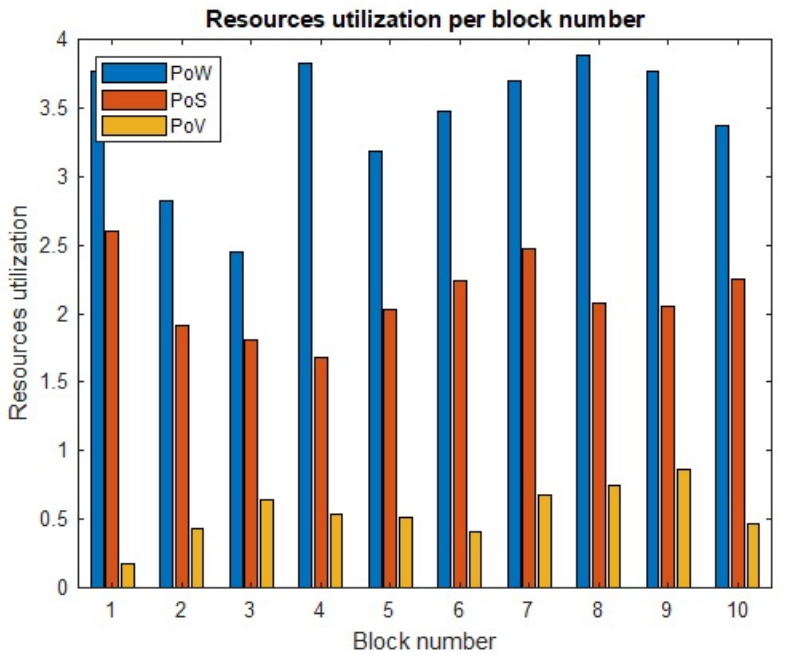

(a) Resources utilization graph for 30 mining nodes

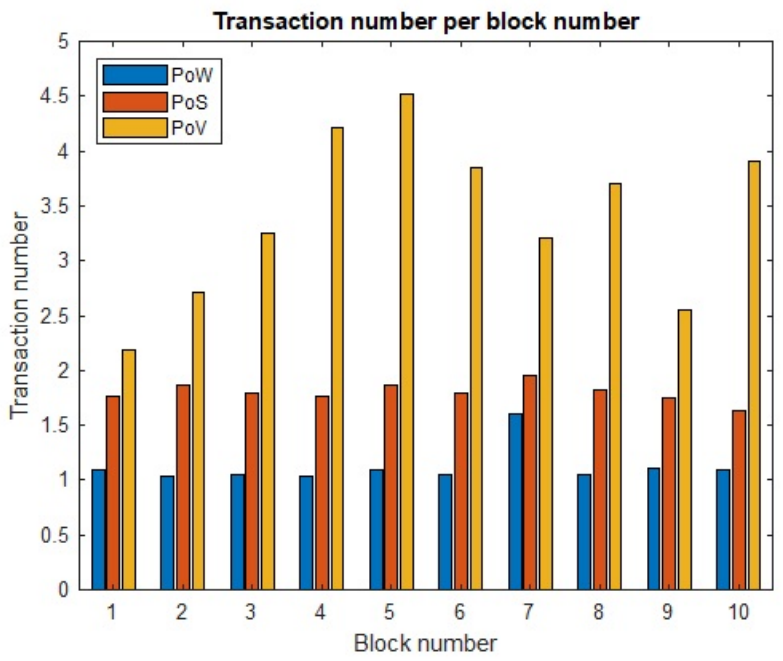

(b) Transaction graph for 30 mining nodes

Fig. 7: Resources utilization and transaction graphs

because the selection of miner in PoV is based on the block stake which is already known to the application when the votes are cast. The block stake is based on a simple probability relating to Blue, Green and Red votes rather than amount of stake owned by individual nodes on the network.

Similarly, Fig. 7b shows the record of transaction numbers per block for the implementation of the three consensus mechanisms: PoW, PoS and PoV. The simulation result shows higher number of transactions per block for PoV than for PoS and higher number of transaction per block for PoS that for PoW. This implies that in PoV, more resources are available for transaction processing than in $\mathrm{PoS}$ and $\mathrm{PoW}$, since PoV uses less energy on consensus than PoS and PoW.

\section{Throughput and Block-time}

The efficiency of the three consensus mechanisms were compared by examining the number of transactions processed with respect to time referred to as throughput. Using the same simulation parameters of 30 mining nodes and 20 million voters on ADEVA, it was observed that the number of transactions 


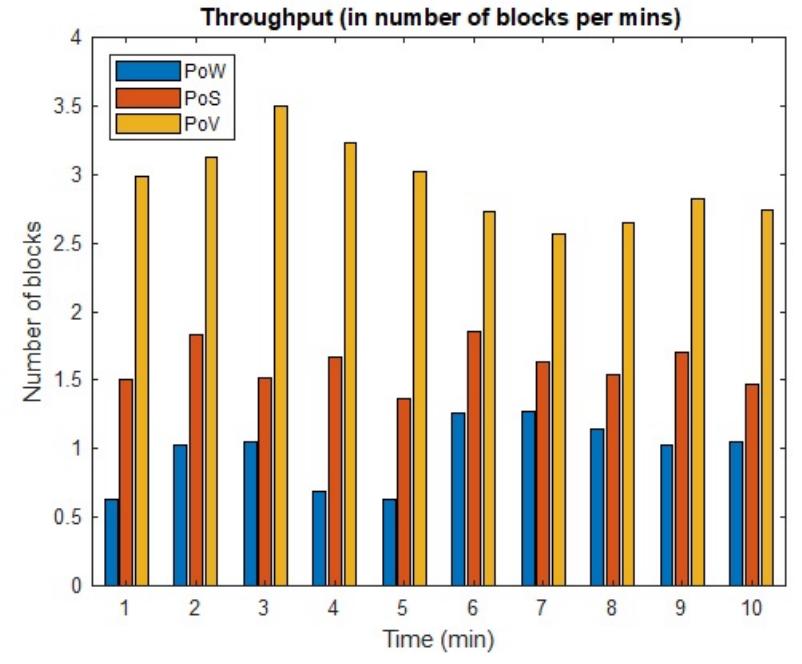

(a) Throughput graph for 30 mining nodes

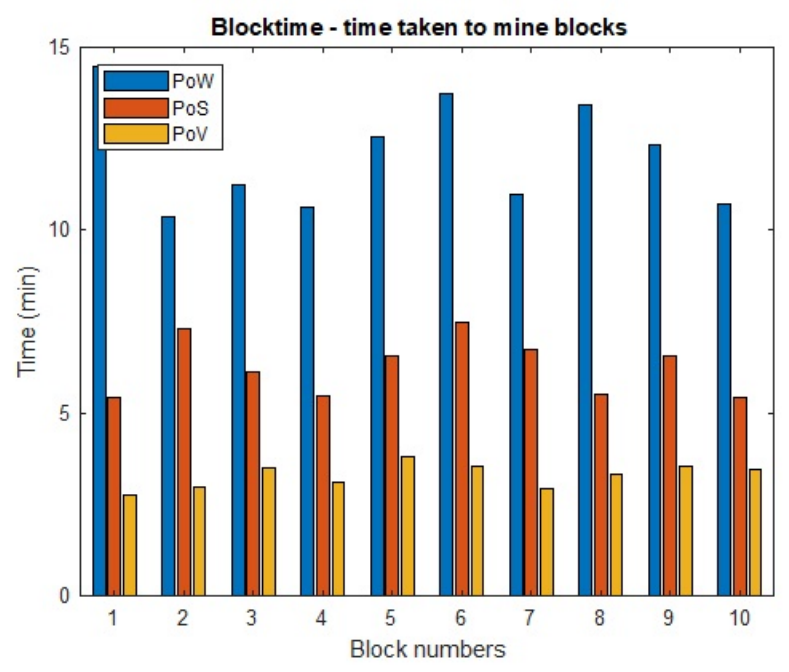

(b) Throughput graph for 30 mining nodes

Fig. 8: Throughput and block-time graphs

per unit time (in mins) is highest for PoV and lowest for PoW (as shown in Fig. 8a).

Similarly, Fig. 8b shows a better performance for PoV when compared with PoS and PoW in terms of block-time which refers to the average time required to mine a block. These observations necessarily follow from the above observations, namely that $\mathrm{PoV}$ process more transactions per unit time for each block than PoS and PoW.

\section{E. Voters Scalability}

In order to test the performance of ADEVA with PoV in handling large numbers of voters (voters' scalability), the number of mining nodes is kept constant at 30 whilst the number of voters is increased gradually from one million to twenty-two million, (the number of registered voters in the state of California, one of the highest numbers of registered voters in the US during 2020, according to the US voters' registration record that is available in the public domain) [58].

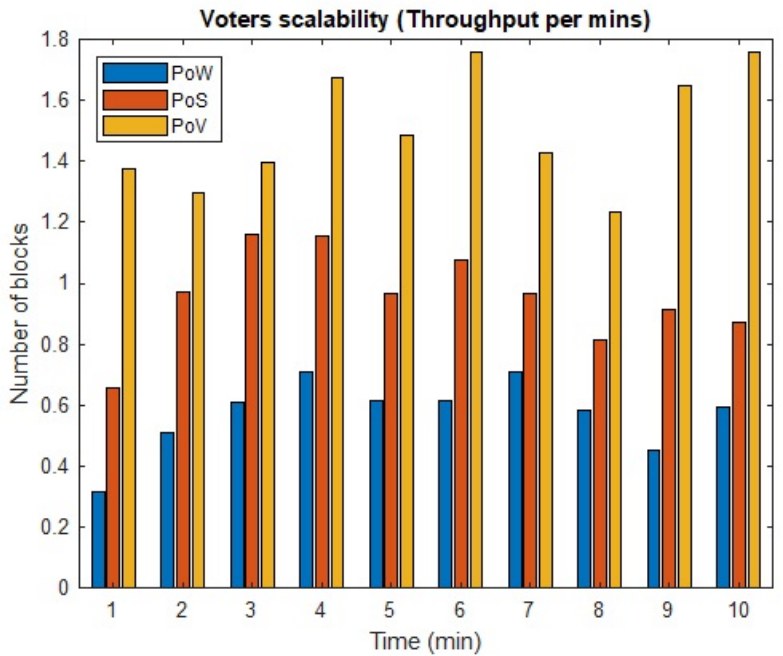

(a) Throughput graph for $22 \mathrm{M}$ voters, 30 mining nodes

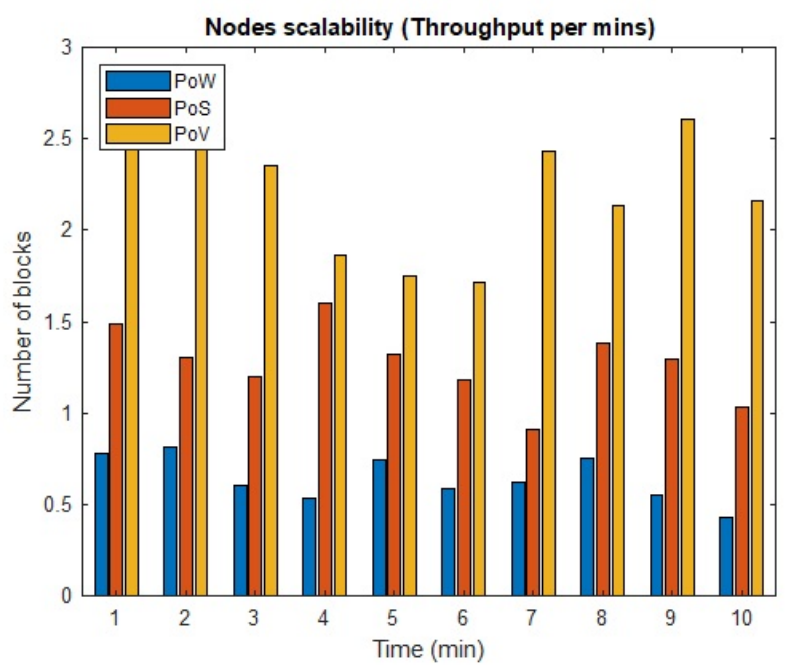

(b) Throughput graph for $22 \mathrm{M}$ voters, 60 mining nodes Fig. 9: Voters and nodes scalability graphs

The casting of votes for this simulation, occurred at random within a 24 hour voting window to accommodate different voting times. The application performance was observed during the scenario. The result (shown in Fig. 9b) shows a better performance for ADEVA with the PoV mechanism than for ADEVA with either the PoS or PoW mechanism.

The simulation was further run with 50 million voters under the same conditions. It was observed that the number of voters arriving at different times during the voting window had no effect on the performance of ADEVA since it takes just a few seconds to cast a vote after a successful MFA. The votes cast were counted on the fly and kept in memory pools whilst awaiting mining.

\section{F. Mining Nodes-Scalability}

In order the test the scalability of nodes under the three consensus mechanisms: PoV, PoS and PoW, ADEVA was simulated with the three consensus mechanisms under the same conditions stated in the subsection V-E above by keeping 
the number of voters constant at twenty-two million whilst increasing the number of mining nodes from three by multiple of three up to sixty. (i.e 3, 6, 9, .. 60). The performance statistics for ADEVA with each of the consensus mechanisms were studied for different numbers of mining nodes shared equally by the three political parties. The results shown in Fig. 9b reflect a better performance for ADEVA simulated with PoV than for ADEVA simulated with either PoS or PoW regardless of the number of nodes used in the simulation runs.

In this simulation run, it was observed that increasing the number of mining nodes had no effect on the application response time, however, the number of blocks generated per unit time (throughput) decreased slightly with an increase in the number of nodes. The result however, still conveys a better throughput for ADEVA with PoV than for ADEVA with PoS or PoW, which both suffer from an increased communication overhead as the number of nodes is increased.

\section{G. ADEVA Cost of Ownership}

The cost of ownership of ADEVA with PoV, is similar to ADEVA with PoS or PoW and it includes the time cost of development and implementation. Such cost is higher than the cost of developing a centralized version of the application because of the need for an Ethereum IDE and the coding of election functionalities into the smart contract.

In addition to this, the overhead of the processing power and communication could make ADEVA more expensive to implement and run with the PoW consensus mechanisms which is the main reason for the proposal of $\mathrm{PoV}$ which is a lightweight consensus mechanism. The potential scalability issue is addressed by dividing the election into smaller chunks known as constituencies, each of which has independent blockchain networks and run a different instance of the application.

\section{MODEL DISCUSSION}

\section{A. Universal Verifiability}

In ADEVA, with the permission of all the election stakeholders, observers could have their monitoring nodes set up as part of the blockchain network to verify transactions. Such monitoring nodes are non-mining nodes that receive all transactions, validate them and thus have a universal copy of the ledger record of e-votes as a verified copy.

\section{B. Voter Verifiability}

A voter will need a TIN and the original hash value of their vote in order to verify the vote. However, in order to satisfy the receipt-free requirements of EVA, this information is not provided to the voters, but stored in the application as part of their voter information or profile. A voter can verify their vote by accessing the application with their login credential and selecting the vote verification option.

This process locates the transaction in the blockchain ledger using the unique TIN associated with the voter and compares the current hash value of the transaction (vote) with the original hash value of the vote stored in the voter's profile and then produce an output message as either 'Vote Unaltered' if they match or 'Vote Altered' if they differ.

This verification process does not reveal the voter's information, neither does it reveal the candidate or party of choice in the vote. The 'Vote Unaltered' message will not be a convincing evidence for a vote buyer, but it will prove to the voters that their votes have not been altered, without revealing the value (or the content) of the vote. Under this condition, in which voting information (the party or candidate of choice) is not revealed during verification, a coercer will, we believe, not be motivated to verify the way that a voter has voted.

\section{Coercion Resistance}

In ADEVA, voting is 'non-geographical' which means that a voter could cast their votes from any location using their own smart devices. We have assumed that a coercer cannot be in all places at all times, to coerce during the voting window. Therefore, for an honest majority of actors and a relatively small number of coercers who cannot be in all places at all times, the coercive effect will be negligible. In order to further frustrate the effort of a coercer, ADEVA is designed to allow repeated voting as a means to deceive coercers, whilst only counting the first vote cast.

In the repeated voting scenario, ADEVA counts the first vote and ignores subsequent ones. This is based on the assumption that voters will cast their votes before the arrival of coercers and the fact that recasting of votes is prohibited in a paper ballot system. Thus, the inclusion of this dummy feature is to enable a voter to cast as many false votes as may be required by different coercers. The assumption also takes care of a scenario in which voters may be forced to hand over their voting credentials to a coercer who could then reuse them at the end of the voting window to overwrite any previous vote.

\section{Receipt Freeness}

The intent of the receipt-free feature is to prevent voters from using their receipt to prove the way they voted to a coercer or a vote buyer. In ADEVA, the TIN and the hash of the votes contains no information to confirm the identity of the voter or the candidate (party) of choice to a vote buyer or a coercer. The TIN and the hash value of the votes are not sent to the voter but are kept in the application as part of the voter's profile for future verification of their vote after voting. This implies that voters will have to login into the application in order to verify their votes. Therefore, ADEVA is receipt-free at the point of voting and the verification procedure prevents vote buying and swaying since it does not reveal the content of the vote. A coercer could log in as a voter (with the voters credentials) to verify votes, but such coercer will be unable to determine how the voter voted because the verification process is a comparison of original hash value of vote with the current hash values of vote without revealing the voters vote.

\section{E. Vote Recast}

Vote recast has its positives and negatives. One of the positives is the flexibility in terms of voters changing their 
minds after casting their votes. The other positive of recast is that it could be used to deceive a coercer. However, a predominant challenge of this feature is the process of deciding which of the multiple votes to tally and count. The two major approaches are: counting the first vote or counting the last vote. If the first vote is cast and counted before the arrival of a coercer, then it is a done deal. The effort of the coercers to coerce will be unsuccessful even if the voting credentials are handed over to them. This approach is implemented in ADEVA. The downside of this approach is that it removes the flexibility that could enable voters to change their mind and vote.

If the last vote is tallied and counted, this enables the voters to be able to change their minds as many times as possible, however, it leaves the them vulnerable to multiple coercive attacks to override the previous votes. Some aggressive coercers could collect the users' voting credentials and use them at the final minutes of the voting window in order to override any previously cast vote. Therefore, on the balance of this risk assessment, counting the first vote and ignoring the subsequent ones will serve as a disincentive to coercers, hence its usage in ADEVA. This implies that for a coercive attack to be successful, coercers will need to do it at the beginning of the voting process which thus limits their operational window.

\section{CONCLUSION AND FUTURE WORK}

EVA has demonstrated huge potential as a replacement for current paper ballot systems. However, some significant considerations such as transparency, receipt-freeness and trustworthiness coupled with vulnerability to DoS attacks, SPF, and fraudulent record modification remain as challenges when evaluating such applications. The proposal of a decentralised application on a blockchain platform as potential solution also has it own challenges relating to throughput, scalability, energy efficiency and thus sustainability, as a result of the PoW consensus mechanism.

In response to these challenges and vulnerabilities, we proposed and developed ADEVA, using an Ethereum Virtual Machine as a decentralized application platform. We also proposed PoV, a new consensus mechanism for ADEVA which we implemented and evaluated with PoV comparing it to ADEVA with PoW and ADEVA with PoS. ADEVA demonstrated an improved performance in terms of transaction speed, throughput, scalability, energy efficiency and thus sustainability. Our future work will involve exploring ADEVA under real life deployment with the intention of fine-tuning its features for improved performance.

\section{REFERENCES}

[1] Kanika Garg, Pavi Saraswat, Sachin Bisht, Sahil Kr. Aggarwal, Sai Krishna Kothuri, and Sahil Gupta. A comparitive analysis on e-voting system using blockchain. In 2019 4th International Conference on Internet of Things: Smart Innovation and Usages (IoT-SIU), pages 1-4, 2019.

[2] Valentin Sliusar, Aleksei Fyodorov, Aleksandr Volkov, Pyotr Fyodorov, and Vladislav Pascari. Blockchain technology application for electronic voting systems. In 2021 IEEE Conference of Russian Young Researchers in Electrical and Electronic Engineering (ElConRus), pages 2257-2261, 2021.
[3] Nir Kshetri and Jeffrey Voas. Blockchain-enabled e-voting. IEEE Software, 35(4):95-99, 2018.

[4] Wenbin Zhang, Yuan Yuan, Yanyan Hu, Shaohua Huang, Shengjiao Cao, Anuj Chopra, and Sheng Huang. A privacy-preserving voting protocol on blockchain. In 2018 IEEE 11th International Conference on Cloud Computing (CLOUD), pages 401-408, 2018.

[5] Wei-Jr Lai, Yung-chen Hsieh, Chih-Wen Hsueh, and Ja-Ling Wu. Date: A decentralized, anonymous, and transparent e-voting system. In 2018 1st IEEE International Conference on Hot Information-Centric Networking (HotICN), pages 24-29, 2018.

[6] Samuel Agbesi and George Asante. Electronic voting recording system based on blockchain technology. In 2019 12th CMI Conference on Cybersecurity and Privacy (CMI), pages 1-8, 2019.

[7] Kriti Patidar and Swapnil Jain. Decentralized e-voting portal using blockchain. In 2019 10th International Conference on Computing, Communication and Networking Technologies (ICCCNT), pages 1-4, 2019.

[8] Basit Shahzad and Jon Crowcroft. Trustworthy electronic voting using adjusted blockchain technology. IEEE Access, 7:24477-24488, 2019.

[9] Shiyao Gao, Dong Zheng, Rui Guo, Chunming Jing, and Chencheng Hu. An anti-quantum e-voting protocol in blockchain with audit function. IEEE Access, 7:115304-115316, 2019.

[10] Kristián Košt'ál, Rastislav Bencel, Michal Ries, and Ivan Kotuliak. Blockchain e-voting done right: Privacy and transparency with public blockchain. In 2019 IEEE 10th International Conference on Software Engineering and Service Science (ICSESS), pages 592-595, 2019.

[11] Deni Pramulia and Bayu Anggorojati. Implementation and evaluation of blockchain based e-voting system with ethereum and metamask. In 2020 International Conference on Informatics, Multimedia, Cyber and Information System (ICIMCIS), pages 18-23, 2020.

[12] Jaewon Bae and Hyuk Lim. Random mining group selection to prevent 51 In 2018 48th Annual IEEE/IFIP International Conference on Dependable Systems and Networks Workshops (DSN-W), pages 8182, 2018.

[13] Gang Sun, Miao Dai, Jian Sun, and Hongfang Yu. Voting-based decentralized consensus design for improving the efficiency and security of consortium blockchain. IEEE Internet of Things Journal, 8(8):62576272, 2021.

[14] Emanuele Bellini, Paolo Ceravolo, and Ernesto Damiani. Blockchainbased e-vote-as-a-service. In 2019 IEEE 12th International Conference on Cloud Computing (CLOUD), pages 484-486, 2019.

[15] Hsin-Te Wu and Chang-Yi Yang. A blockchain-based network security mechanism for voting systems. In 2018 1st International Cognitive Cities Conference (IC3), pages 227-230, 2018.

[16] Ashish Singh and Kakali Chatterjee. Secevs : Secure electronic voting system using blockchain technology. In 2018 International Conference on Computing, Power and Communication Technologies (GUCON), pages 863-867, 2018.

[17] Y. Abuidris, A. Hassan, A. Hadabi, and I. Elfadul. Risks and opportunities of blockchain based on e-voting systems. In 2019 16th International Computer Conference on Wavelet Active Media Technology and Information Processing, pages 365-368, 2019.

[18] Nedaa Baker Al Barghuthi, Issam Hamdan, Saleh Al Suwaidi, Ahmed Lootah, Bader Al Amoudi, Omar Al Shamsi, and Saeed Al Aryani. An analytical view on political voting system using blockchain technology - uae case study. In 2019 Sixth HCT Information Technology Trends (ITT), pages 132-137, 2019.

[19] Ali Mansour Al-madani, Ashok T. Gaikwad, Vivek Mahale, and Zeyad A.T. Ahmed. Decentralized e-voting system based on smart contract by using blockchain technology. In 2020 International Conference on Smart Innovations in Design, Environment, Management, Planning and Computing (ICSIDEMPC), pages 176-180, 2020.

[20] Amit Kumar Tyagi, Terrance Frederick Fernandez, and S U Aswathy. Blockchain and aadhaar based electronic voting system. In 2020 4th International Conference on Electronics, Communication and Aerospace Technology (ICECA), pages 498-504, 2020.

[21] R. Han, V. Gramoli, and X. Xu. Evaluating blockchains for iot. In 2018 9th IFIP International Conference on New Technologies, Mobility and Security (NTMS), pages 1-5, Feb 2018.

[22] Yikang Lin and Peng Zhang. Blockchain-based complete self-tallying evoting protocol. In 2019 Asia-Pacific Signal and Information Processing Association Annual Summit and Conference (APSIPA ASC), pages 47$52,2019$. 
[23] Feng Hao, Matthew N. Kreeger, Brian Randell, Dylan Clarke, Siamak F. Shahandashti, and Peter Hyun-Jeen Lee. Every vote counts: Ensuring integrity in large-scale electronic voting. USENIX Journal of Election Technology and Systems (JETS), (3):1-25, August 2014.

[24] F. Hao, P. Y. A. Ryan, and P. Zieliński. Anonymous voting by two-round public discussion. IET Information Security, 4(2):62-67, 2010.

[25] Sudharsan B, Rishi Tharun V, Nidhish Krishna M P, Boopathi Raj J, Surya Arvindh M, and Dr. M. Alagappan. Secured electronic voting system using the concepts of blockchain. In 2019 IEEE 10th Annual Information Technology, Electronics and Mobile Communication Conference (IEMCON), pages 0675-0681, 2019.

[26] Wei Cai, Zehua Wang, Jason B. Ernst, Zhen Hong, Chen Feng, and Victor C. M. Leung. Decentralized applications: The blockchainempowered software system. IEEE Access, 6:53019-53033, 2018.

[27] Dalia Khader, Ben Smyth, Peter Y. A. Ryan, and Feng Hao. A fair and robust voting system by broadcast. In Manuel J. Kripp, Melanie Volkamer, and Rüdiger Grimm, editors, 5th International Conference on Electronic Voting 2012 (EVOTE2012), pages 285-299, Bonn, 2012. Gesellschaft für Informatik e.V.

[28] Lakshmi Priya K., M.Naveen Kumar Reddy, and L. Maruthi Manohar Reddy. An integrated and robust evoting application using private blockchain. In 2020 4th International Conference on Trends in Electronics and Informatics (ICOEI)(48184), pages 842-846, 2020.

[29] David Khoury, Elie F. Kfoury, Ali Kassem, and Hamza Harb. Decentralized voting platform based on ethereum blockchain. In 2018 IEEE International Multidisciplinary Conference on Engineering Technology (IMCET), pages 1-6, 2018.

[30] Abhishek Kaudare, Milan Hazra, Anurag Shelar, and Manoj Sabnis. Implementing electronic voting system with blockchain technology. In 2020 International Conference for Emerging Technology (INCET), pages $1-9,2020$.

[31] Freya Sheer Hardwick, Apostolos Gioulis, Raja Naeem Akram, and Konstantinos Markantonakis. E-voting with blockchain: An e-voting protocol with decentralisation and voter privacy. In 2018 IEEE International Conference on Internet of Things (iThings) and IEEE Green Computing and Communications (GreenCom) and IEEE Cyber, Physical and Social Computing (CPSCom) and IEEE Smart Data (SmartData), pages 1561-1567, 2018.

[32] R. Hanifatunnisa and B. Rahardjo. Blockchain based e-voting recording system design. In 2017 11th International Conference on Telecommunication Systems Services and Applications (TSSA), pages 1-6, 2017.

[33] Erick Febriyanto, Triyono, Nina Rahayu, Kelvin Pangaribuan, and Po Abas Sunarya. Using blockchain data security management for evoting systems. In 2020 8th International Conference on Cyber and IT Service Management (CITSM), pages 1-4, 2020.

[34] R. A. Canessane, N. Srinivasan, A. Beuria, A. Singh, and B. M Kumar. Decentralised applications using ethereum blockchain. In 2019 Fifth International Conference on Science Technology Engineering and Mathematics (ICONSTEM), volume 1, pages 75-79, 2019.

[35] Patrick McCorry, Siamak F. Shahandashti, and Feng Hao. A smart contract for boardroom voting with maximum voter privacy. In Aggelos Kiayias, editor, Financial Cryptography and Data Security, pages 357 375, Cham, 2017. Springer International Publishing.

[36] M. Soud, S. Helgason, G. Hjálmtýsson, and M. Hamdaqa. Trustvote: On elections we trust with distributed ledgers and smart contracts. In 2020 2nd Conference on Blockchain Research Applications for Innovative Networks and Services (BRAINS), pages 176-183, 2020.

[37] Archit Pandey, Mohit Bhasi, and K. Chandrasekaran. Votechain: A blockchain based e-voting system. In 2019 Global Conference for Advancement in Technology (GCAT), pages 1-4, 2019.

[38] T.M. Roopak and R Sumathi. Electronic voting based on virtual id of aadhar using blockchain technology. In 2020 2nd International Conference on Innovative Mechanisms for Industry Applications (ICIMIA), pages $71-75,2020$

[39] Fáber D. Giraldo, Barbosa Milton C., and Carlos E. Gamboa. Electronic voting using blockchain and smart contracts: Proof of concept. IEEE Latin America Transactions, 18(10):1743-1751, 2020.

[40] Aaron Fernandes, Karan Garg, Ankit Agrawal, and Ashutosh Bhatia Decentralized online voting using blockchain and secret contracts. In 2021 International Conference on Information Networking (ICOIN), pages 582-587, 2021.

[41] Ehab Zaghloul, Tongtong Li, and Jian Ren. d-bame: Distributed blockchain-based anonymous mobile electronic voting. IEEE Internet of Things Journal, pages 1-1, 2021.
[42] Vijayalakshmi V. and Vimal S. A novel p2p based system with blockchain for secured voting scheme. In 2019 Fifth International Conference on Science Technology Engineering and Mathematics (ICONSTEM), volume 1, pages 153-156, 2019.

[43] Cosmas Krisna Adiputra, Rikard Hjort, and Hiroyuki Sato. A proposal of blockchain-based electronic voting system. In 2018 Second World Conference on Smart Trends in Systems, Security and Sustainability (WorldS4), pages 22-27, 2018.

[44] K Teja, MB Shravani, Chintarlapallireddy Yaswanth Simha, and Manjunath R Kounte. Secured voting through blockchain technology. In 2019 3rd International Conference on Trends in Electronics and Informatics (ICOEI), pages 1416-1419, 2019.

[45] Chinnapong Angsuchotmetee, Pisal Setthawong, and Sapjarern Udomviriyalanon. Blockvote : An architecture of a blockchain-based electronic voting system. In 2019 23rd International Computer Science and Engineering Conference (ICSEC), pages 110-116, 2019.

[46] Steve A. Adeshina and Adegboyega Ojo. Maintaining voting integrity using blockchain. In 2019 15th International Conference on Electronics, Computer and Computation (ICECCO), pages 1-5, 2019.

[47] Silvia Bartolucci, Pauline Bernat, and Daniel Joseph. Sharvot: Secret share-based voting on the blockchain. In 2018 IEEE/ACM 1st International Workshop on Emerging Trends in Software Engineering for Blockchain (WETSEB), pages 30-34, 2018.

[48] Donny Seftyanto, Amiruddin Amiruddin, and Arif Rahman Hakim. Design of blockchain-based electronic election system using hyperledger: Case of indonesia. In 2019 4th International Conference on Information Technology, Information Systems and Electrical Engineering (ICITISEE), pages 228-233, 2019.

[49] Mohamed Ibrahim, Kajan Ravindran, Hyon Lee, Omair Farooqui, and Qusay H. Mahmoud. Electionblock: An electronic voting system using blockchain and fingerprint authentication. In 2021 IEEE 18th International Conference on Software Architecture Companion (ICSAC), pages 123-129, 2021.

[50] Emre Yavuz, Ali Kaan Koç, Umut Can Çabuk, and Gökhan Dalkılıç. Towards secure e-voting using ethereum blockchain. In 2018 6th International Symposium on Digital Forensic and Security (ISDFS), pages $1-7,2018$.

[51] Awsan A. H. Othman, Emarn A. A. Muhammed, Haneen K. M. Mujahid, Hamzah A. A. Muhammed, and Mogeeb A. A. Mosleh. Online voting system based on iot and ethereum blockchain. In 2021 International Conference of Technology, Science and Administration (ICTSA), pages $1-6,2021$.

[52] Amish Khandelwal. Blockchain implimentation on e-voting system. In 2019 International Conference on Intelligent Sustainable Systems (ICISS), pages 385-388, 2019.

[53] Kejiao Li, Hui Li, Hanxu Hou, Kedan Li, and Yongle Chen. Proof of vote: A high-performance consensus protocol based on vote mechanism amp; consortium blockchain. In 2017 IEEE 19th International Conference on High Performance Computing and Communications; IEEE 15th International Conference on Smart City; IEEE 3rd International Conference on Data Science and Systems (HPCC/SmartCity/DSS), pages 466-473, 2017.

[54] Raphael Matile, Bruno Rodrigues, Eder Scheid, and Burkhard Stiller. Caiv: Cast-as-intended verifiability in blockchain-based voting. In 2019 IEEE International Conference on Blockchain and Cryptocurrency (ICBC), pages 24-28, 2019.

[55] Sankarshan Damle, Sujit Gujar, and Moin Hussain Moti. Fasten: Fair and secure distributed voting using smart contracts. In 2021 IEEE International Conference on Blockchain and Cryptocurrency (ICBC), pages 1-3, 2021.

[56] Shalini Shukla, A.N. Thasmiya, D.O. Shashank, and H.R. Mamatha. Online voting application using ethereum blockchain. In 2018 International Conference on Advances in Computing, Communications and Informatics (ICACCI), pages 873-880, 2018.

[57] Yamuna Rosasooria, Abd Kadir Mahamad, Sharifah Saon, Mohd Anuar Mat Isa, Shingo Yamaguchi, and Mohd Anuaruddin Ahmadon. Evoting on blockchain using solidity language. In 2020 Third International Conference on Vocational Education and Electrical Engineering (ICVEE), pages 1-6, 2020.

[58] Enrique Mafla and Cesar Gallardo. Numerical analysis of ecuador's electoral register integrity. In 2018 International Conference on eDemocracy eGovernment (ICEDEG), pages 351-355, 2018. 